\title{
On the Boundedness of Solutions of Discontinuous Differential Equations
}

\author{
I.L.D. SANTOS
}

Received on July 9, 2020 / Accepted on February 15, 2021

\begin{abstract}
By using the notion of Carathéodory solution to differential equations, the present work studies the boundedness of solutions of discontinuous differential equations. For these discontinuous systems determined by discontinuous differential equations, results are obtained that guarantee sufficient conditions to boundedness of solutions in terms of nonsmooth Lyapunov functions.
\end{abstract}

Keywords: boundedness of solutions, discontinuous differential equations, Carathéodory solutions.

\section{INTRODUCTION}

Ordinary differential equations with the discontinuous right side, also called discontinuous differential equations, determine discontinuous systems. The boundedness of solutions to discontinuous systems determined by autonomous differential equations was studied by [2].

Motivated by the study of the boundedness of solutions for ordinary differential equations in terms of smooth Lyapunov functions (see for instance [9] and [15]), this report studies the boundedness of solutions of discontinuous systems determined by nonautonomous differential equations.

The study carried out here makes use of the notion of Carathéodory solution for discontinuous differential equations. In addition, nonpathological functions are used as Lyapunov functions.

Qualitative results via nonsmooth Lyapunov functions for solutions to discontinuous differential equations can be found, for example, in $[3,7,12,13]$. By using the notion of Filippov solution, [13] studies Lyapunov stability of equilibria of discontinuous systems. On the other hand, [3], [12] and [7] address Lyapunov stability of equilibria of discontinuous systems by using the notion of Carathéodory solution.

The discontinuous systems studied in this work are determined by

$$
\dot{x}(t)=f(t, x(t))
$$

Departament of Mathematics, Sao Paulo State University, Ilha Solteira, SP, Brazil - E-mail: iguer.santos@unesp.br https://orcid.org/0000-0001-5237-6024 
and by

$$
\dot{x}(t)=g(x(t))
$$

where $f: \mathbb{R} \times \mathbb{R}^{n} \rightarrow \mathbb{R}^{n}$ and $g: \mathbb{R}^{n} \rightarrow \mathbb{R}^{n}$. For the study of the boundedness of solutions of the system (1.1) and (1.2), it is assumed throughout the work that for every $x_{0} \in \mathbb{R}^{n}$, each of the differential equations given in (1.1) and (1.2) admit at least one Carathéodory solution with the initial condition $x\left(t_{0}\right)=x_{0}$. It is also assumed that all Carathéodory solutions to (1.1) and (1.2) satisfying $x\left(t_{0}\right)=x_{0}$, are defined on the interval $\left[t_{0},+\infty\right)$. The existence of Carathéodory solutions for (1.1) has been treated in [5] and [8]. On the other hand, a study on the continuation of solutions can be found in [14].

Thus, in the present study results are established (Theorems 3.1, 3.2, 3.3 and 3.4) that provide sufficient conditions for the boundedness of solutions of (1.1) and (1.2) in terms of nonsmooth Lyapunov functions. Theorems 3.1 and 3.3 establish results for the boundedness of solutions of (1.1), while Theorems 3.2 and 3.4 establish results for the boundedness of solutions of (1.2).

\section{PRELIMINARIES}

In this section are considered basic concepts and results that will be used throughout the work.

\subsection{Carathéodory solution}

Solutions to the differential equation (1.1) will be understood as absolutely continuous functions. A approach to absolutely continuous functions can be found in [10]. Below, the concept of absolutely continuous function on an interval $[a, b]$ is defined.

Definition 2.1. A function $x:[a, b] \rightarrow \mathbb{R}^{n}$ is said to be absolutely continuous if for any $\varepsilon>0$, there exists $\delta>0$ such that, for any countable collection of disjoint subintervals $\left[a_{k}, b_{k}\right]$ of $[a, b]$ obeying

$$
\sum\left(b_{k}-a_{k}\right)<\delta
$$

implies that

$$
\sum\left|x\left(b_{k}\right)-x\left(a_{k}\right)\right|<\varepsilon .
$$

Let $I \subset \mathbb{R}$ be an interval. It is said that a statement $P$ holds almost everywhere (a.e.) on $I$, if the set $N$ given by

$$
N=\{t \in I: P \text { does not hold at } t\}
$$

has Lebesgue measure zero. A more complete approach to Lebesgue measure can be found in [11].

Definition 2.2. Let $I \subset \mathbb{R}$ be an interval. A function $x: I \rightarrow \mathbb{R}^{n}$ is a Carathéodory solution of (1.1) on I if $x(t)$ is absolutely continuous and $\dot{x}(t)=f(t, x(t))$ for a.e. $t \in I$. 
Consider, for example, the following discontinuous differential equation

$$
\dot{x}(t)=\operatorname{sgn}(t)
$$

where $\operatorname{sgn}(\cdot)$ is the sign function, that is,

$$
\operatorname{sgn}(t)= \begin{cases}1, & t>0 \\ 0, & t=0 \\ -1, & t<0 .\end{cases}
$$

As discussed in [1], the Carathéodory solution of (2.1) that satisfies the condition $x(0)=c$ is given by $x(t)=|t|+c$.

\subsection{Nonpathological functions}

The following is an approach on nonpathological functions. The considerations made here on nonpathological functions are based on [3].

In the following, $\partial V(x)$ denotes Clarke's generalized gradient for the function $V(x)$ at $x$. See [4] for a better approach to generalized gradients.

Definition 2.3. Let $V: \mathbb{R}^{n} \rightarrow \mathbb{R}$ be a locally Lipschitz function. The generalized gradient of $V$ at $x$ is defined by

$$
\partial V(x)=\overline{c o}\left\{\lim \nabla V\left(x_{i}\right) \mid x_{i} \rightarrow x, x_{i} \notin \Omega_{V}\right\}
$$

where $\overline{c o}$ denotes the closure of the convex hull, and $\Omega_{V}$ is the set of measure zero (in the sense of Lebesgue measure) such that the gradient of $V$ is not defined.

From the previous definition, if $V: \mathbb{R} \times \mathbb{R}^{n} \rightarrow \mathbb{R}$ is given by $V(t, x)=V_{1}(x)$, where $V_{1}: \mathbb{R}^{n} \rightarrow \mathbb{R}$, then $\partial V(t, x)=\left(0, \partial V_{1}(x)\right)$.

Definition 2.4. It is said that a function $V: \mathbb{R}^{n} \rightarrow \mathbb{R}$ is nonpathological if it is locally Lipschitz continuous and for every absolutely continuous function $\varphi: I \subset \mathbb{R} \rightarrow \mathbb{R}^{n}$ and for a.e. $t \in I$, the set $\partial V(\varphi(t))$ is a subset of an affine subspace orthogonal to $\dot{\varphi}(t)$.

The proposition stated below can be founded in [3].

Proposition 2.1. If $V: \mathbb{R}^{n} \rightarrow \mathbb{R}$ is nonpathological, and $\varphi: \mathbb{R} \rightarrow \mathbb{R}^{n}$ is absolutely continuous, then the set $\{p \cdot \dot{\varphi}(t): p \in \partial V(\varphi(t))\}$ is reduced to the singleton $\left\{\frac{d}{d t} V(\varphi(t))\right\}$ for a.e. $t$.

Nonpathological derivative is defined below.

Definition 2.5. Let $V: \mathbb{R} \times \mathbb{R}^{n} \rightarrow \mathbb{R}$ be a nonpathological function and let $A_{V}$ be the set given by

$$
A_{V}=\left\{(t, x) \in \mathbb{R} \times \mathbb{R}^{n}: p_{1} \cdot(1, f(t, x))=p_{2} \cdot(1, f(t, x)), \forall p_{1}, p_{2} \in \partial V(t, x)\right\} .
$$

If $(t, x) \in A_{V}$, the nonpathological derivative of the map $V$ with respect to (1.1) at $(t, x)$ is the number

$$
\dot{\bar{V}}_{f}(t, x)=p \cdot(1, f(t, x))
$$


where $p$ is any vector in $\partial V(t, x)$.

From Proposition 2.1 follows the following corollary.

Corollary 2.0. Consider the nonpathological function $V: \mathbb{R} \times \mathbb{R}^{n} \rightarrow \mathbb{R}$, and let $\varphi$ be any solution of (1.1). Then $(t, \varphi(t)) \in A_{V}$ and $\frac{d}{d t} V(t, \varphi(t))=\dot{\bar{V}}_{f}(t, \varphi(t))$ for a.e. $t$.

In [3], nonpathological functions $V: \mathbb{R}^{n} \rightarrow \mathbb{R}$ are used to study the autonomous system (1.2). In this case, the set $A_{V}$ is defined as

$$
A_{V}=\left\{x \in \mathbb{R}^{n}: p_{1} \cdot g(x)=p_{2} \cdot g(x), \forall p_{1}, p_{2} \in \partial V(x)\right\} .
$$

If $x \in A_{V}$, the nonpathological derivative of the map $V$ with respect to (1.2) at $x$ is defined by

$$
\dot{\bar{V}}_{g}(x)=p \cdot g(x)
$$

where $p$ is any vector in $\partial V(x)$.

The next corollary is stated in [3].

Corollary 2.0. Let the function $V: \mathbb{R}^{n} \rightarrow \mathbb{R}$ be nonpathological, and let $\varphi$ be any solution of system (1.2). Then $\varphi(t) \in A_{V}$ and $\frac{d}{d t} V(\varphi(t))=\dot{\bar{V}}_{g}(\varphi(t))$ for a.e. $t$.

\section{BOUNDEDNESS OF SOLUTIONS}

In what follows are obtained the main results of the work, the Theorems 3.1, 3.2, 3.3 and 3.4. Below are defined the concepts of boundedness that are used in the present work.

Suppose that the set of Carathéodory solutions of (1.1) with initial condition $x\left(t_{0}\right)=x_{0}$ is denoted by $S\left(x_{0}, t_{0}\right)$. Also suppose that for any initial conditions $\left(x_{0}, t_{0}\right)$ the set $S\left(x_{0}, t_{0}\right)$ is nonempty and all solutions are defined on the interval $\left[t_{0}, \infty\right)$. If $x(t) \in S\left(x_{0}, t_{0}\right)$, the notation $x\left(t, t_{0}, x_{0}\right)$ will also be used to denote $x(t)$.

Definition 3.6. A solution $x(t) \in S\left(x_{0}, t_{0}\right)$ is bounded if there exists a $\beta>0$ such that $|x(t)|<\beta$ for $t \geq t_{0}$, where $\beta$ may depend on each solution.

Definition 3.7. The solutions of (1.1) are uniformly bounded if for any $\alpha>0$ and $t_{0} \geq 0$, there is a $\beta=\beta(\alpha)>0$ (independent of $t_{0}$ ) such that $\left|x_{0}\right|<\alpha$ implies $|x(t)|<\beta$ for $t \geq t_{0}$ and $x(t) \in S\left(x_{0}, t_{0}\right)$.

Definition 3.8. The solutions of (1.1) are uniformly ultimately bounded (with bound $L$ ) if there exists $a L>0$ and if for any $\alpha>0$ and any $t_{0} \geq 0$, there is a $T=T(\alpha)>0$ (independent of $t_{0}$ ) such that $\left|x_{0}\right|<\alpha$ implies that $|x(t)|<L$ for $t \geq t_{0}+T$ and $x(t) \in S\left(x_{0}, t_{0}\right)$.

Similarly, the boundedness of solutions for (1.2) is defined.

It can be seen that the solutions of (2.1) are not bounded, or even uniformly bounded. 
Theorems 3.1 and 3.3 and their proofs are similar to their smooth counterparts. The smooth version of Theorem 3.1 can be founded in [ [9], Theorem 9.13] and [ [15], Theorem 10.2]. On the other hand, the smooth version of Theorem 3.3 can be founded in [ [9], Theorem 9.14] and [ [15], Theorem 10.4]. For completeness and ease of reading, the proofs of Theorems 3.1 and 3.3 will be played.

Before stating Theorems 3.1, 3.2, 3.3 and 3.4, the definitions of the functions of class $\mathscr{K}$ and class $\mathscr{K} \mathscr{R}$ are considered.

Definition 3.9. It is said that a continuous function $\psi:\left[0, r_{1}\right] \rightarrow[0, \infty)$ (respectively, $\psi:[0, \infty) \rightarrow$ $[0, \infty)$ ) belongs to class $\mathscr{K}$, that is, $\psi \in \mathscr{K}$, if $\psi(0)=0$ and if $\psi$ is strictly increasing on $\left[0, r_{1}\right]$ (respectively, on $[0, \infty)$ ). It is said that a function $\psi \in \mathscr{K}$ definided on $[0, \infty)$ belongs to class $\mathscr{K} \mathscr{R}$ if $\lim _{r \rightarrow \infty} \psi(r)=+\infty$.

Theorem 3.1. Let $V: \mathbb{R} \times \mathbb{R}^{n} \rightarrow \mathbb{R}$ be a nonpathological function and let $R>0$. Suppose that there exist functions $\psi_{1}$ and $\psi_{2} \in \mathscr{K} \mathscr{R}$ satisfying

$$
\psi_{1}(|x|) \leq V(t, x) \leq \psi_{2}(|x|)
$$

for all $|x| \geq R$ and all $t \geq 0$. If $\dot{\bar{V}}_{f}(t, x) \leq 0$ for all $(t, x) \in A_{V}$ with $|x| \geq R$ and $t \geq 0$, then the solutions of (1.1) are uniformly bounded.

Proof. Take $k>R$ and consider $\left(t_{0}, x_{0}\right) \in[0, \infty) \times \mathscr{B}(k)$ with $\left|x_{0}\right|>R$, where $\mathscr{B}(k)$ is the open ball of radius $k$ centered at the origin. Now, let $\phi_{0}(t) \in S\left(x_{0}, t_{0}\right)$ be denoted by $\phi\left(t, t_{0}, x_{0}\right)$ and define $v(t)$ by $v(t)=V\left(t, \phi_{0}(t)\right)$ for as long as $\left|\phi_{0}(t)\right|>R$. Since $v(t)$ is an absolutely continuous function,

$$
v(t)=v\left(t_{0}\right)+\int_{t_{0}}^{t} \dot{v}(s) d s .
$$

From Corollary 2.0, $\dot{\bar{V}}_{f}\left(t, \phi_{0}(t)\right)=\frac{d}{d t} V\left(t, \phi_{0}(t)\right)$ for a.e. $t$, and using the hypothesis $\dot{\bar{V}}_{f}(t, x) \leq 0$, it can be concluded that $v(t) \leq v\left(t_{0}\right)$. Hence,

$$
\psi_{1}\left(\left|\phi_{0}(t)\right|\right) \leq v(t) \leq v\left(t_{0}\right) \leq \psi_{2}\left(\left|x_{0}\right|\right) \leq \psi_{2}(k) .
$$

Since $\psi_{1} \in \mathscr{K} \mathscr{R}$, its inverse exists and $\left|\phi_{0}(t)\right| \leq \beta:=\psi_{1}^{-1}\left(\psi_{2}(k)\right)$ for as long as $\left|\phi_{0}(t)\right|>R$.

If $\left|\phi_{0}(t)\right|$ starts at a value smaller than $R$ or if it reaches a value less than $R$ for some $t>t_{0}$, then $\phi_{0}(t)$ can remain in $\mathscr{B}(k)$ for all subsequent $t$ or else it may leave $\mathscr{B}(k)$ on a interval $t_{1}<t<t_{2} \leq+\infty$. On the interval $I=\left(t_{1}, t_{2}\right)$, the aforementioned argument yields $\left|\phi_{0}(t)\right| \leq \beta$ on $I$. Therefore $\left|\phi_{0}(t)\right| \leq \max \{R, \beta\}$ for all $t \geq t_{0}$.

It is also possible to state a result analogous to the Theorem 3.1 for systems determined by autonomous differential equations.

Theorem 3.2. Let $V: \mathbb{R}^{n} \rightarrow \mathbb{R}$ be a nonpathological function and let $R>0$. Suppose that there exist functions $\psi_{1}$ and $\psi_{2} \in \mathscr{K} \mathscr{R}$ obeying

$$
\psi_{1}(|x|) \leq V(x) \leq \psi_{2}(|x|)
$$


for all $|x| \geq R$. If $\dot{\bar{V}}_{g}(x) \leq 0$ for all $x \in A_{V}$ with $|x| \geq R$, then the solutions of (1.2) are uniformly bounded.

Theorem 3.3. Let $V: \mathbb{R} \times \mathbb{R}^{n} \rightarrow \mathbb{R}$ be a nonpathological function and let $R>0$. Suppose that there exist functions $\psi_{1}, \psi_{2} \in \mathscr{K} \mathscr{R}$ such that

$$
\psi_{1}(|x|) \leq V(t, x) \leq \psi_{2}(|x|)
$$

for all $|x| \geq R$ and all $t \geq 0$. If in addition, there exists a function $\psi_{3} \in \mathscr{K}$ satisfying $\dot{\bar{V}}_{f}(t, x) \leq$ $-\psi_{3}(|x|)$ for all $(t, x) \in A_{V}$ with $|x| \geq R$ and $t \geq 0$, then the solutions of (1.1) are uniformly ultimately bounded.

Proof. Consider $k_{1}>R$ and let $B>k_{1}$ be such that $\psi_{2}\left(k_{1}\right)<\psi_{1}(B)$. This is possible because $\psi_{1} \in$ $\mathscr{K} \mathscr{R}$. Take $k_{2}>B$ and let $T=\left[\psi_{2}\left(k_{2}\right) / \psi_{3}\left(k_{1}\right)\right]+1$. With $B<\left|x_{0}\right| \leq k_{2}$ and $t_{0} \geq 0$, let $\phi_{0}(t)=$ $\phi\left(t, t_{0}, x_{0}\right) \in S\left(x_{0}, t_{0}\right)$ and $v(t)=V\left(t, \phi_{0}(t)\right)$. Since $v(t)$ is a absolutely continuous function,

$$
v(t)=v\left(t_{0}\right)+\int_{t_{0}}^{t} \dot{v}(s) d s .
$$

It follows from Corollary 2.0 that $\dot{\bar{V}}_{f}\left(t, \phi_{0}(t)\right)=\frac{d}{d t} V\left(t, \phi_{0}(t)\right)$ for a.e. $t$, and then

$$
v(t)=v\left(t_{0}\right)+\int_{t_{0}}^{t} \dot{\bar{V}}_{f}\left(s, \phi_{0}(s)\right) d s .
$$

The function $\left|\phi_{0}(t)\right|$ must satisfy $\left|\phi_{0}\left(t^{*}\right)\right| \leq k_{1}$ for some $t^{*} \in\left(t_{0}, t_{0}+T\right)$, otherwise, suppose that $\left|\phi_{0}(t)\right|>k_{1}$ for all $t \in\left(t_{0}, t_{0}+T\right)$. It follows from hypothesis $\dot{\bar{V}}_{f}(t, x) \leq-\psi_{3}(|x|)$ that

$$
\begin{aligned}
v(t) & =v\left(t_{0}\right)+\int_{t_{0}}^{t} \dot{\bar{V}}_{f}\left(s, \phi_{0}(s)\right) d s \leq v\left(t_{0}\right)-\int_{t_{0}}^{t} \psi_{3}\left(\left|\phi_{0}(s)\right|\right) d s \\
& \leq \psi_{2}\left(\left|x_{0}\right|\right)-\int_{t_{0}}^{t} \psi_{3}\left(\left|\phi_{0}(s)\right|\right) d s \leq \psi_{2}\left(k_{2}\right)-\int_{t_{0}}^{t} \psi_{3}\left(k_{1}\right) d s \\
& =\psi_{2}\left(k_{2}\right)-\psi_{3}\left(k_{1}\right)\left(t-t_{0}\right) .
\end{aligned}
$$

Hence, if $t=T+t_{0}$,

$$
0 \leq \psi_{1}\left(\left|\phi_{0}\left(T+t_{0}\right)\right|\right) \leq v\left(T+t_{0}\right) \leq \psi_{2}\left(k_{2}\right)-\psi_{3}\left(k_{1}\right)(T)=-\psi_{3}\left(k_{1}\right)<0
$$

So, $t^{*}$ must exist.

Now, suppose that $\left|\phi_{0}\left(t^{*}\right)\right|=k_{1}$ and $\left|\phi_{0}(t)\right|>k_{1}$ for $t \in\left(t^{*}, t_{1}\right)$, where $t_{1} \leq+\infty$. Since $v(t)$ is nonincreasing in $t$,

$$
\psi_{1}\left(\left|\phi_{0}(t)\right|\right) \leq v(t) \leq v\left(t^{*}\right) \leq \psi_{2}\left(\left|\phi_{0}\left(t^{*}\right)\right|\right)=\psi_{2}\left(k_{1}\right)<\psi_{1}(B)
$$

for all $t \geq t^{*}$. Thus, $\left|\phi_{0}(t)\right|<B$ for all $t \geq t^{*}$.

Below is a result similar to the Theorem 3.3 for systems determined by autonomous differential equations. 
Theorem 3.4. Let $V: \mathbb{R}^{n} \rightarrow \mathbb{R}$ be a nonpathological function and let $R>0$. Suppose that there exist functions $\psi_{1}, \psi_{2} \in \mathscr{K} \mathscr{R}$ such that

$$
\psi_{1}(|x|) \leq V(x) \leq \psi_{2}(|x|)
$$

for all $|x| \geq R$. If in addition, there exists a function $\psi_{3} \in \mathscr{K}$ obeying $\dot{\bar{V}}_{g}(x) \leq-\psi_{3}(|x|)$ for all $x \in A_{V}$ with $|x| \geq R$, then the solutions of (1.2) are uniformly ultimately bounded.

As an example for the use of Theorem 3.2, consider the Artstein's circles example

$$
\left\{\begin{array}{l}
\dot{x}=\left(x^{2}-y^{2}\right) u \\
\dot{y}=2 x y u
\end{array}\right.
$$

where $u \in \mathbb{R}$. In the same way that [ [3], Example 1], let

$$
u(x, y)= \begin{cases}1, & x<0 \\ -1, & x \geq 0\end{cases}
$$

Let $V: \mathbb{R}^{n} \rightarrow \mathbb{R}$ be the nonpathological function $V(x, y)=\sqrt{4 x^{2}+3 y^{2}}-|x|$. Take $(x, y) \in A_{V}$ arbitrary and consider $\dot{\bar{V}}_{g}(x, y)$, where $g(x, y)=\left(\left(x^{2}-y^{2}\right) u, 2 x y u\right)$. If $x \neq 0$, then $\partial V(x, y)=$ $\nabla V(x, y)$. Thence $\dot{\bar{V}}_{g}(x, y) \leq 0$, since

$$
\nabla V(x, y) \cdot g(x, y)=\frac{-4|x|^{3}-2|x| y^{2}+\left(x^{2}-y^{2}\right) \sqrt{4 x^{2}+3 y^{2}}}{\sqrt{4 x^{2}+3 y^{2}}} \leq 0
$$

whenever $x \neq 0$. Otherwise, suppose that $x=0$. If $y>0$,

$$
\left(0, \frac{3}{\sqrt{3}}\right) \in \partial V(0, y)=\left\{\left(s, \frac{3}{\sqrt{3}}\right): s \in[-1,1]\right\}
$$

and if $y<0$,

$$
\left(0,-\frac{3}{\sqrt{3}}\right) \in \partial V(0, y)=\left\{\left(s,-\frac{3}{\sqrt{3}}\right): s \in[-1,1]\right\} .
$$

In its turn, if $y=0$ then

$$
(0,0) \in \partial V(0,0)=\left\{(s, v): s \in[-1,1], v \in\left[-\frac{3}{\sqrt{3}}, \frac{3}{\sqrt{3}}\right]\right\} .
$$

Anyways, if $x=0$ it follows that $g(x, y)=\left(-y^{2} u, 0\right)$ and so

$$
\dot{\bar{V}}_{g}(x, y)=0 \text {. }
$$

Hence $\dot{\bar{V}}_{g}(x, y) \leq 0$ for all $(x, y) \in A_{V}$. From Theorem 3.2 the solutions of (3.1) are uniformly bounded.

Now, for the use of Theorem 3.1, it will be considered a system of differential equations treated by [7]. In this way, consider the two dimensional system

$$
\left\{\begin{array}{l}
\dot{x}=-x f_{2}(t, x, y)+|y| \\
\dot{y}=-y f_{1}(t, x, y)+|x|
\end{array}\right.
$$


where $f_{1}, f_{2}:[0, \infty) \times \mathbb{R}^{2} \rightarrow \mathbb{R}, f_{2}(t, x, y) \geq \operatorname{sgn}(y)$ and $f_{1}(t, x, y) \geq \operatorname{sgn}(x)$. For the application of Theorem 3.1, consider the function $V: \mathbb{R} \times \mathbb{R}^{2} \rightarrow \mathbb{R}$ given by $V(t, x, y)=V_{1}(x, y)$, where $V_{1}(x, y)=|x|+|y|$ is nonpathological. Take $(t, x, y) \in A_{V}$ arbitrary. Hence,

$$
\begin{aligned}
\dot{\bar{V}}_{f}(t, x, y) & =\bigcap_{\xi \in \partial V(t, x, y)} \xi \cdot(1, f(t, x, y)) \\
& =\bigcap_{\xi \in\left(0, \partial V_{1}(x, y)\right)} \xi \cdot(1, f(t, x, y)) \\
& =\bigcap_{\eta \in \partial V_{1}(x, y)} \eta \cdot f(t, x, y)
\end{aligned}
$$

where $f(t, x, y)=\left(-x f_{2}(t, x, y)+|y|,-y f_{1}(t, x, y)+|x|\right)$. If $x \neq 0$ and $y \neq 0$, it follows that $\partial V(t, x, y)=\left(0, \nabla V_{1}(x, y)\right)=(0, \operatorname{sgn}(x), \operatorname{sgn}(y))$. Consequently $\dot{\bar{V}}_{f}(t, x, y) \leq 0$, since

$$
\begin{aligned}
& (0, \operatorname{sgn}(x), \operatorname{sgn}(y)) \cdot(1, f(t, x, y)) \\
& =\operatorname{sgn}(x)\left(-x f_{2}(t, x, y)+|y|\right)+\operatorname{sgn}(y)\left(-y f_{1}(t, x, y)+|x|\right) \\
& =-|x| f_{2}(t, x, y)+\operatorname{sgn}(x)|y|-|y| f_{1}(t, x, y)+\operatorname{sgn}(y)|x| \\
& =-|x|\left(f_{2}(t, x, y)-\operatorname{sgn}(y)\right)-|y|\left(f_{1}(t, x, y)-\operatorname{sgn}(x)\right) \leq 0 .
\end{aligned}
$$

Now, suppose that $y=0$. If $x>0$,

$$
(1,0) \in \partial V_{1}(x, 0)=\{(1, s): s \in[-1,1]\}
$$

and thus

$$
\begin{aligned}
\dot{\bar{V}}_{f}(t, x, 0) & =(1,0) \cdot\left(-x f_{2}(t, x, 0),|x|\right) \\
& =-x f_{2}(t, x, 0) \leq 0
\end{aligned}
$$

seeing that $f_{2}(t, x, 0) \geq \operatorname{sgn}(0)=0$. If $x<0$,

$$
(-1,0) \in \partial V_{1}(x, 0)=\{(-1, s): s \in[-1,1]\}
$$

and thus

$$
\begin{aligned}
\dot{\bar{V}}_{f}(t, x, 0) & =(-1,0) \cdot\left(-x f_{2}(t, x, 0),|x|\right) \\
& =x f_{2}(t, x, 0) \leq 0 .
\end{aligned}
$$

On the other hand, suppose that $x=0$. If $y>0$,

$$
(0,1) \in \partial V_{1}(0, y)=\{(s, 1): s \in[-1,1]\}
$$

and then

$$
\begin{aligned}
\dot{\bar{V}}_{f}(t, 0, y) & =(0,1) \cdot\left(|y|,-y f_{1}(t, 0, y)\right) \\
& =-y f_{1}(t, 0, y) \leq 0
\end{aligned}
$$


because $f_{1}(t, 0, y) \geq \operatorname{sgn}(0)=0$. If $y<0$,

$$
(0,-1) \in \partial V_{1}(0, y)=\{(s,-1): s \in[-1,1]\}
$$

and then

$$
\begin{aligned}
\dot{\bar{V}}_{f}(t, 0, y) & =(0,-1) \cdot\left(|y|,-y f_{1}(t, 0, y)\right) \\
& =y f_{1}(t, 0, y) \leq 0
\end{aligned}
$$

Lastly, if $(x, y)=(0,0)$,

$$
(0,0) \in \partial V_{1}(0,0)=\{(s, v): s \in[-1,1], v \in[-1,1]\}
$$

and then $\dot{\bar{V}}_{f}(t, 0,0)=0$. Whence $\dot{\bar{V}}_{f}(t, x, y) \leq 0$ for all $(t, x, y) \in A_{V}$, and from Theorem 3.1 the solutions of (3.2) are uniformly bounded.

Next, a fashion equation arising from the Physics of Fluids in the past 30 years is considered. More specifically, consider Eq. (6.2) of paper [6] with $N=2$,

$$
\left\{\begin{array}{l}
\dot{p}_{j}=2 \sum_{k=1}^{2} p_{j} p_{k} \operatorname{sgn}\left(q_{j}-q_{k}\right) e^{-\left|q_{j}-q_{k}\right|} \\
\dot{q}_{j}=\sum_{k=1}^{2} p_{k} e^{-\left|q_{j}-q_{k}\right|}
\end{array}\right.
$$

where $j \in\{1,2\}$. The Eq. (6.2) of [6] is a discontinuous differential equation that is widely studied. Note that Theorems 3.2 and 3.4 do not apply to (3.3), since $p_{1}(t) \equiv 0, p_{2}(t) \equiv 1, q_{1}(t)=t$ and $q_{2}(t)=t$ provide a solution not bounded to (3.3).

\section{CONCLUSIONS}

The work contributes to the qualitative theory of discontinuous systems. More specifically, the work studies the boundedness of solutions to discontinuous systems determined by discontinuous differential equations. The boundedness results established here refer to the concepts of solutions uniformly bounded and solutions uniformly ultimately bounded. The main results of the work are stated in the Theorems 3.1, 3.2, 3.3 and 3.4. Theorems 3.1 and 3.3 establish boundedness results to systems determined by nonautonomous differential equations, while Theorems 3.2 and 3.4 establish boundedness results to systems determined by autonomous differential equations.

\section{REFERENCES}

[1] K.A. Alshammari. "Filippov's operator and discontinuous differential equations". Ph.D. thesis, Louisiana State University and Agricultural and Mechanical College, Baton Rouge, LA (2006).

[2] A.F. Armaki, N. Pariz \& R. Asgharian. Construction of nonpathological Lyapunov functions for discontinuous systems with Carathéodory solutions. Asian J Control, 14(1) (2012), 284-287.

[3] A. Bacciotti \& F. Ceragioli. Nonpathological Lyapunov functions and discontinuous Carathéodory systems. Automatica, 42(3) (2006), 453-458. 
[4] F.H. Clarke. "Optimization and nonsmooth analysis". Canadian Mathematical Society Series of Monographs and Advanced Texts. John Wiley \& Sons, Inc., New York (1983).

[5] E.A. Coddington \& N. Levinson. "Theory of ordinary differential equations". McGraw-Hill Book Company, Inc., New York-Toronto-London (1955).

[6] A. Degasperis, D.D. Holm \& A.N.W. Hone. A new integrable equation with peakon solutions. Theor Math Phys, 133(2) (2002), 1463-1474.

[7] M. Grzanek, A. Michalak \& A. Rogowski. A nonsmooth Lyapunov function and stability for ODE's of Carathéodory type. Nonlinear Anal, 69(1) (2008), 337-342.

[8] J.K. Hale. "Ordinary differential equations”. Robert E. Krieger Publishing Co., Inc., Huntington, N.Y., second ed. (1980).

[9] R.K. Miller \& A.N. Michel. "Ordinary differential equations". Academic Press, New York (1982).

[10] I.P. Natanson. "Theory of functions of a real variable". Frederick Ungar Publishing Co., New York (1955).

[11] H.L. Royden. "Real analysis". The Macmillan Co., New York (1963).

[12] I.L.D. Santos. Lyapunov stability for discontinuous systems. Ciência e Natura, 42 (2020), 1-08.

[13] D. Shevitz \& B. Paden. Lyapunov stability theory of nonsmooth systems. IEEE Trans Automat Control, 39(9) (1994), 1910-1914.

[14] P.G. Stechlinski \& P.I. Barton. Dependence of solutions of nonsmooth differential-algebraic equations on parameters. J Differential Equations, 262(3) (2017), 2254-2285.

[15] T. Yoshizawa. "Stability theory by Liapunov's second method". The Mathematical Society of Japan, Tokyo (1966). 